\title{
Vasil Grigoriev
}

Independent researcher. Belarus

\section{Causes and mechanism of development of periodontitis and caries. The relationship between periodontal diseases, depression and orofacial pain.}

Background \& Aim

GRRGV dental system is a system of means and methods that solve the problem of preventing periodontal disease and caries. The basis element of the GRRGV system was found in 1991. Since 1991 the GRRGV dental system has been developing to the present. The term "periodontal depression" is introduced to refer to the period when there are no symptoms of PDC, but in the tooth there are neural and vascular disorders that lead to the appearance of symptoms PDC. GRRGV dental system solves the problem of preventing nerve and vascular disorders in the teeth.

The problem of periodontal disease and caries.

It is believed that periodontitis is difficult to treat and it is impossible to completely cure. Many dentists believe that periodontitis is incurable. The exact cause of periodontitis is unknown. Gingivitis in dentistry is considered to be transitional to periodontitis. After the course of treatment of gingivitis, the inflammatory process occurs later on again. As a cause of periodontitis, the periodontal lesion of plaque bacteria and the violation of the periodontal blood supply are considered. Periodontitis is on the third place after incurable after cardiology and oncology. The causes of periodontal disease and caries (PDC) are bacterial damage to the teeth by plaque bacteria.The problem of methods for the treatment of periodontitis.

The problem of methods for the treatment of periodontitis.

Methods of treating periodontitis are based on various assumptions, under conditions when the main cause of periodontitis is unknown. There are a lot of methods for treating periodontitis, but gingivitis and periodontitis is present in $100 \%$ of people over 40 years old. In 20-25 years or earlier symptoms of gingivitis and periodontitis may appear and further progression of periodontitis. When periodontitis, parodontium is actively affected by bacteria and therefore various measures are being developed to control the bacterial lesion of periodontal disease. 
Research is carried out on the properties of microorganisms present in the oral cavity and methods of their destruction. But the methods of antibiotic therapy used in combination with surgical methods of treatment are ineffective. The search for solutions is based on the use of various combinations of immune and antioxidant drugs, surgical treatment and other methods of controlling bacterial infection. All these methods of treatment do not completely eliminate the symptoms of periodontal disease. After a possible improvement, there is an exacerbation and they are used when all the symptoms of periodontitis are already present and do not provide full recovery. There may be temporary changes in the direction of improvement, but they do not solve the problem of periodontitis. No means of complete curing or prevention of periodontitis have been found. Therefore, there is a need for fundamentally new methods for monitoring periodontal diseases. And since after damage periodontium is not restored, the main goal is to prevent the appearance of periodontitis.

The search for the causes of periodontitis.

The mechanism of periodontal disease is not defined. There are many theories of the onset of periodontitis. Why do many different in their nature local and common factors lead to periodontitis? From this follows the assumption of the existence of one generalized cause of periodontitis. Numerous different nature factors may not be the cause of BP. According to WHO statistics, almost all people are affected by periodontal diseases and these diseases progress despite the wide use of various methods for treating periodontal diseases. Studies on the problem of treatment of BP do not bring progress on this problem. And the actual detailed study of periodontal pathologies does not give a result and does not provide effective methods for solving problems with periodontium. This situation is possible if the study of the BP problem does not take into account the main factor, which is the generalized cause of problems with periodontium. The inefficiency of existing methods is often explained by the appearance of qualitatively new pathogenic microorganisms or other causes. Attempts to solve the BP problem are developing in the direction of increasing complexity of the methods of treatment and involving in them an increasing number of systems and means.

Periodontal depression is the cause of symptoms of periodontitis and caries.

The primary cause of periodontal diseases and caries (hereinafter PDC) is periodontal depression (hereinafter DEP) - a pathological condition, which in most 
cases occurs quickly - within a month. PDC have a common cause of occurrence, which is DEP. The destructive changes occurring in the tooth due to the effects of bacteria are due to the state of the DEP. In the absence of DEP in the tooth, inflammatory and atrophic processes do not occur. DEP precedes the period of appearance in the tooth of destructive reversible or irreversible changes. The state of DEP is the cause of the appearance of PDC symptoms. DEP is characterized by neurovascular disorders in the tooth, which lead to atrophic and inflammatory processes in the tooth. DEP is the initial stage of the PDC. Symptoms of DEP are depressive disorders, clinical depression and orofacial pain and other symptoms. If the state of the DEP is not eliminated, then symptoms of PDC arise. The GRRGV dental system eliminates DEP and provides a tooth condition at which PDC do not occur. The GRRGV dental system (hereinafter GRRGV system) helps to eliminate gum disease and ensures the absence of inflammation in the future. Present carious lesions under the condition of using the GRRGV dental system do not progress. As a preventive measure, the GRRGV dental system ensures that there are no symptoms of PDC for the entire period of application of the GRRGV dental system. The GRRGV dental system solves the problem of treating vascular and nerve disorders in the tooth. The practice of applying the GRRGV system proves that caries and periodontal diseases can be avoided. And also proves the common causes of caries and periodontal diseases. GRRGV system eliminates the common cause of PDC - periodontal depression, The GRRGV system normalizes the vascular and nerve processes in the tooth and eliminates stagnation in the teeth. PDC arise as a consequence of several factors - accumulation of stagnant phenomena in the teeth, the features of the structure of the tooth, the speed of cell renewal in the periodontium, and nervous and vascular disorders. The mechanism for the development of the destructive process is the rapid renewal of periodontal cells and the impossibility of their complete withdrawal, due to vascular-neural disorders. There is a rapid accumulation of slag cells in the tooth and as a result the tooth is affected by microorganisms. PDC have one overall development pattern and a common primary cause of occurrence. The effectiveness of the GRRGV system is based on the elimination of neurovascular disorders in the tooth. Initially, there are neurovascular disorders in the tooth, and then the tooth is damaged by microorganisms or the tooth is affected by atrophic processes. The GRRGV system provides the basis for dental health for any length of time.

In the state of DEP, nerve and vascular disorders occur in the tooth. At the initial stage of the DEP, the symptoms of PDC are absent. In the periodontium, the cells are constantly renewed. The epithelium of the area of the dentogingival attachment is updated in 4-10 days, the gingival cells are also quickly updated. 
Nervous and vascular disorders in the periodontium occur simultaneously and are closely interrelated. The rapidity of renewal of periodontal cells in combination with DEP lead to chronic congestion in the periodontium. This stagnation occurs very quickly. Already after 4-10 days (the time of renewal of periodontal cells), a favorable environment can be created for the destruction of periodontal microorganisms. There is a situation of continuous deterioration of the periodontium due to the accumulation of a large number of slag cells, which for a period of one month should be excised from the periodontal. The general state of the body affects the periodontal condition, but DEP occurs even in the absence of systemic problems. Symptoms of DEP are depressive disorders, orofacial pain and other symptoms. The internal state of the periodontium is determined using GRRGV signs, which determine the periodontal condition at any time. The GRRGV dental system uses tools to determine the internal state of periodontal disease. The GRRGV system examines the condition of the tooth before the appearance of the symptoms of PDC and prevents the appearance and development of symptoms of PDC. The GRRGV system solves the problem of PDC prophylaxis with the help of special features that allow monitoring the state of the tooth of the teeth during the absence of symptoms of PDC.

Relationship between the state of DEP and depression.

The state of DEP is the source of depressive disorders. DEP is a local factor and affects the general condition of a person. DEP has a systemic effect on the human body. The question is why with good nutrition and a healthy lifestyle, the condition of the teeth still worsens can lead to internal disharmony. With DEP, the processes that occur in the tooth are manifested by depressive disorders and orofacial pain. Daily fixation of tooth decay negatively affects the general mood of a person. The value of a beautiful smile with healthy teeth is high. In the tooth is a large number of nerve fibers. Violations in them affect the overall nervous activity of a person. DEP is a continuously and rapidly emerging state throughout the life of a person. The processes of constant reproduction of the state of DEP arise from a very early age. The condition of periodontal disease is characterized by rapid changes.

General scheme for the development of the PDC.

A distinctive feature of periodontium is the rapidity of the processes occurring in it. Within a period of 4-10 days, periodontal cells are renewed. In the presence of DEP state, stagnant phenomena in the tooth quickly appear, against which the symptoms of PDC are then manifested. For example, for a period of 6 months in the tooth, irreversible destructive processes can occur. The appearance 
of PDC symptoms occurs independently of the general systemic state of the organism. Also, other external favorable factors do not protect against the appearance of PDC symptoms. For example, good nutrition or a healthy external environment also does not protect against the occurrence of symptoms of PDC. Symptoms of PDC arise as a combination of DEP and local physiological characteristics of the tooth. Vascular and nerve disorders lead to stagnation in the tooth. And then there are symptoms of PDC. The treatment of PDC symptoms does not eliminate the main reason - DEP. Therefore, in the future, symptoms of PDC arise again. The GRRGV dental system eliminates the conditions for the occurrence of PDC symptoms. The removal of microorganisms from the oral cavity does not protect teeth from damage by microorganisms. Teeth in the state of DEP can be affected by various types of microorganisms that, in the healthy condition of periodontal disease, do not exhibit pathogenic activity. In the state of DEP, teeth are almost constantly in a state in which they can easily be infected with microorganisms. Destructive phenomena in the tooth against the background of the DEP intensify almost every week. Dental plaque can be a supplier of microorganisms if the tooth is in the DEP state and is susceptible to damage by microorganisms. The condition of the teeth is in a dynamic change. Quickly create favorable conditions for tooth damage by microorganisms. Early detection of PDC symptoms does not lead to a positive result in treatment if the DEP state is not eliminated. The dental system GRRGV provides at this time in 50 years and 10 months the absence of new cases of caries since 1991 and the complete absence of any symptoms of periodontal disease. With a high degree of confidence, we can predict the continuation of this trend.

\section{Fig.1}

Stage 1 Under ordynary conditions, teeth are in a healthy state for 3-10 days.

Stage 2 Periodontal depression occurs in 3-10 days. At the initial stage, there are nervous disorders in the teeth. Then there are vascular disorders in the teeth. Vascular disorders create conditions for the lesion of teeth by bacteria. Depressive disorders and orofacial pain arise. At this stage, clinical depression may occur. Nervous and vascular disorders in teeth lead to the appearance of symptoms of periodontal disease and caries. The GRRGV system makes it possible to determine the condition of the teeth and normalize their condition.

Stage 3 Periodontal depression goes into a chronic stage. Nerve and vascular disorders in the teeth accumulate and lead to the appearance of PDC symptoms. 
Periodontal depression leads to depressive disorders and orofacial pain, that negatively affect a person.

Stage 4 Loss of teeth.

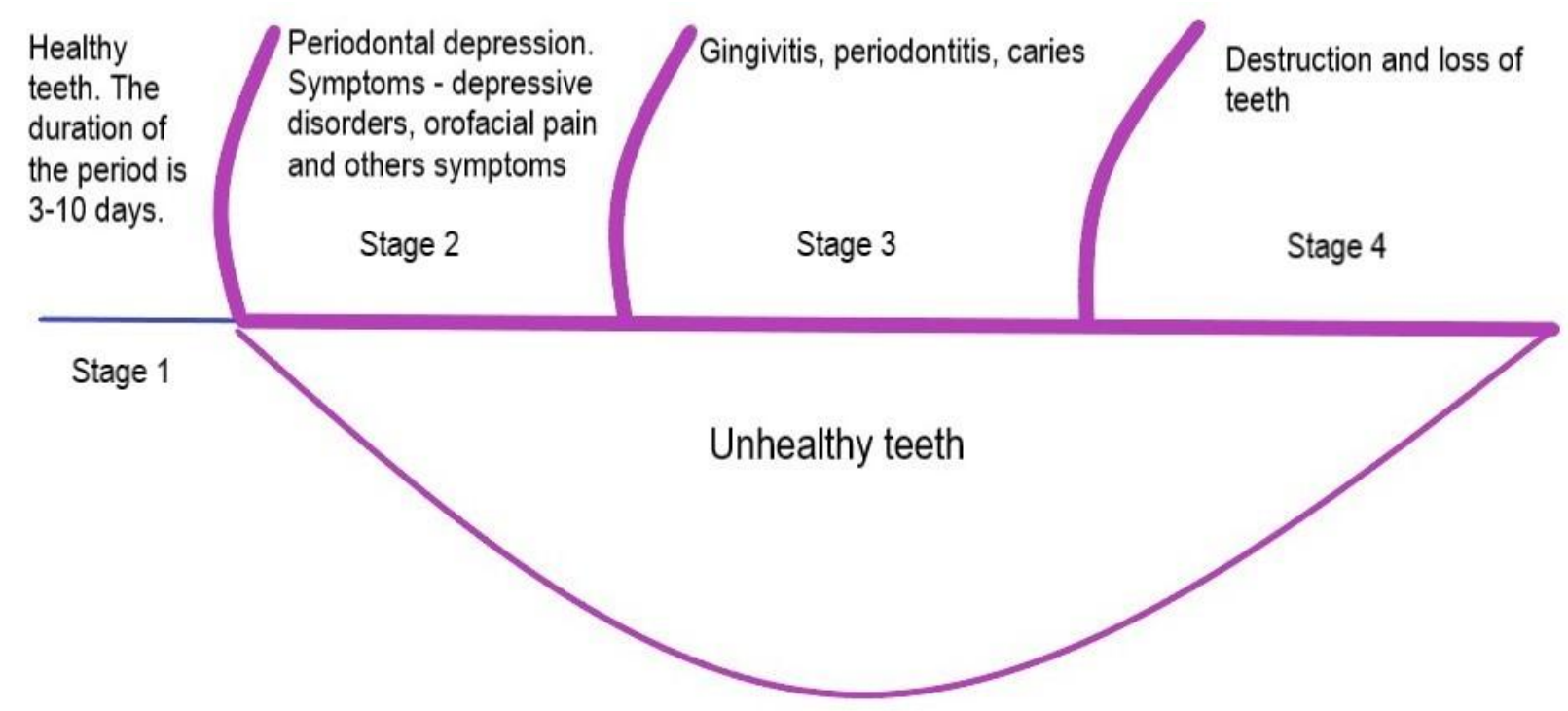

Fig.1

Conclusions

1. Periodontal disease is disease with single local cause. Periodontal depression is the cause of the symptoms of PDC. GRRGV system determines the state of the teeth in a period of periodontal depression and eliminates nerve and vascular disorders. PDEP have systemic effect on the entire human body. PDEP cause disorders in the work of many human organs.

2. Bacterial plaque is not the primary cause of periodontal disease. At first periodontal depression. The composition of plaque is formed under the influence of the periodontal depression. Changing the composition of the biofilm can cause secondary bacterial damage to the teeth, but bacterial plaque is not the primary cause of periodontal disease.

3. The symptom of periodontal depression is major depressive disorder ( depression). For the treatment of depression it is necessary to eliminate the periodontal depression. Depression is a symptom of periodontal depression. The primary cause of periodontal disease is nerve disorders in the teeth. At the same time, there are vascular disorders. The GRRGV system makes it possible to establish that the level of nerve disorders in the teeth is high. 
4. Symptom of periodontal depression is orofacial pain. GRRGV system is a remedy that eliminates orofacial pain completely. The GRRGV system eliminates the nerve disorders in the teeth, which are the cause of orofacial pain. Depression and orofacial pain - symptoms of periodontal depression.

5. GRRGV system is a system tool that provides oral health.The GRRGV system solves the problem of periodontitis, caries, gingivitis, halitosis and other problems. And the GRRGV system solves other problems that are associated with dental health - depressive disorders, orofacial pain and other problems. All these listed problems arise due to the periodontal depression.

6. The problem of dental health is an interdisciplinary problem. Many of the problems that are now considered unresolved (periodontitis, caries, halitosis, orofacial pain, depressive disorders and other problems) have a common cause periodontal depression.

7. Periodontal depression is the cause of tooth decay. Caries and periodontal diseases have single common cause - PDEP. 8. The use of the GRRGV system stops destructive processes in the teeth and normalizes the condition of the teeth. 10 out of 10 over 40 -year-olds suffer from some kind of periodontal disease. The absence of periodontal disease at the age of 50 years is sufficient proof of the effectiveness of the GRRGV system. The initial stage of PDC is PDEP. GRRGV system acts on the cause of PDC and eliminates nerve disorders in the tooth and prevents the appearance of symptoms PDC for a period of more than 25 years.

Fig.2 -3 Age 50 years 7 months. Pictures taken on 04/22/2018. 3 There are small damages to the enamel of the tooth, which were formed due to improper tooth cleaning with a toothbrush. These injuries are invisible. No hypersensitivity to hot, cold, sweet and sour. Teeth with enamel damage behave just like teeth without damaging the enamel.The damage to the teeth that are present is not progressing and does not cause inconvenience. 


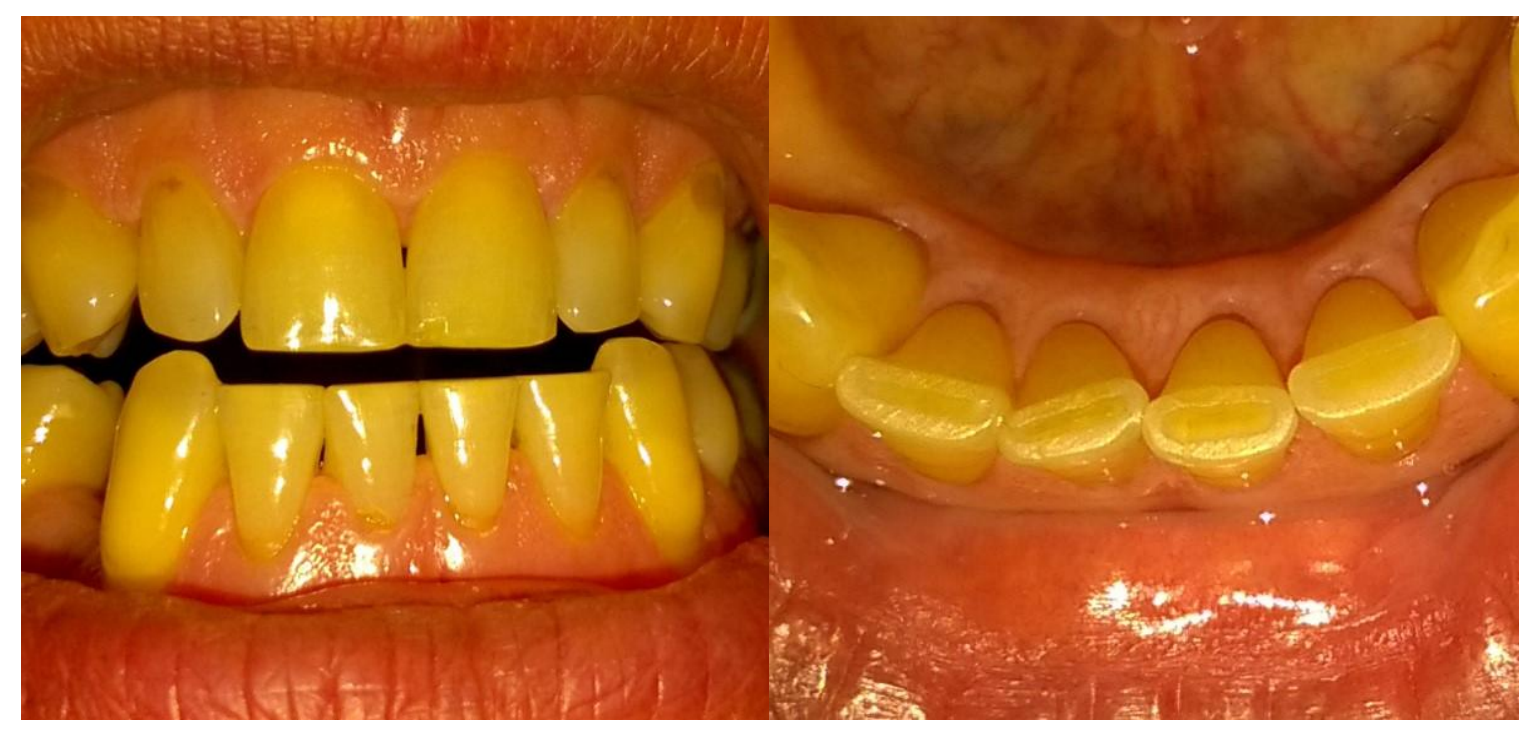

Fig. 2

Fig. 3

References: Vasil Grigoriev. Complete Solution of Problem of Caries Prevention and Periodontal Diseases : Eastern European Scientific Journal, No 3, 2017; 13-18. DOI 10.12851/EESJ201703 